Research Paper

\title{
AKAPII gene polymorphism is associated with bone mass measured by quantitative ultrasound in young adults
}

\author{
María Correa-Rodríguez ${ }^{1}$, Jacqueline Schmidt Rio-Valle², Blanca Rueda-Medina ${ }^{2 \bowtie}$ \\ 1. Assistance Professor, PhD. Faculty of Health Sciences. University of Granada (Spain). \\ 2. Professor, PhD. Faculty of Health Sciences. University of Granada (Spain). \\ $\square$ Corresponding author: Schmidt Rio-Valle Jacqueline; Tel.: +34 958243498; Fax: 958242894; Faculty of Health Sciences. Av. Ilustración, S/N, 18017 Granada \\ (Spain); E-mail address: jschmidt@ugr.es \\ (c) Ivyspring International Publisher. This is an open access article distributed under the terms of the Creative Commons Attribution (CC BY-NC) license \\ (https://creativecommons.org/licenses/by-nc/4.0/). See http://ivyspring.com/terms for full terms and conditions.
}

Received: 2018.02.05; Accepted: 2018.05.27; Published: 2018.06.14

\begin{abstract}
Background: Due to the increased prevalence of osteoporosis and direct health care cost of osteoporosis-related fractures, there is a growing interest in identifying genetic markers associated with osteoporosis phenotypes in order to develop genetic screening strategies. We aimed to analyze the possible associations between calcaneal Quantitative ultrasound (QUS), a valuable screening tool for assessing bone status in clinical practice, and ZBTB40 (rs7524102, rs6426749), SP7 (rs2016266) and AKAPII (rs9533090) genes.
\end{abstract}

Methods: A cross-sectional study was conducted on 550 healthy individuals of Caucasian ancestry ( 381 females and 169 males, median age $20.46 \pm 2,69)$. Bone mass was assessed through QUS to determine broadband ultrasound attenuation (BUA, dB/MHz). Single-nucleotide polymorphisms (SNPs) in ZBTB40 (rs7524102, rs6426749), SP7 (rs2016266) and AKAPI I (rs9533090) were selected as genetic markers and genotyped using TaqMan OpenArray ${ }^{\circledR}$ technology.

Results: Linear regression analysis revealed that rs7524102 and rs6426749 in ZBTB40, and rs9533090 in AKAPII were significantly associated with the calcaneal QUS parameter after adjustments for age, sex, weight, height, physical activity, and calcium intake $(p=0.038, p=0.012$ and $p=0.008$, respectively). After applying the Bonferroni correction for multiple testing $(p=0.012)$, only the association of $r s 9533090$ in AKAPII remained significant.

Conclusion: AKAPI I gene (rs9533090) influences QUS trait in a population of Caucasian young adults. The rs 9533090 SNP may be considered a factor affecting peak bone mass acquisition.

Key words: polymorphism; AKAP11 gene; bone mass; young adults; quantitative ultrasound.

\section{Introduction}

Osteoporosis, the most common chronic metabolic bone disease, is a systemic skeletal disorder characterized by low bone mass and microarchitectural deterioration of bone tissue, with a consequent increase in bone fragility and susceptibility to fractures [1]. It is considered a global epidemic since it has been estimated than more than 200 million people are suffering from osteoporosis [2]. In addition, osteoporosis is associated with decreased quality of life and increased disability-adjusted life span [3, 4]. Moreover, it implies a significant health care cost to insurance systems of countries [5]. Osteoporosis is determined by the effects of several genes, which exert a relatively modest effects, and modifiable variables such diet and physical activity [6].

Advances in the knowledge of the genetics of osteoporosis will allow individual genetic profiles to become a common routine practice, facilitating more personalized health care [7]. Moreover, genetic screening will be increasingly relevant in the disease prevention and early treatment [8]. Due to the increased prevalence of osteoporosis and direct health 
care cost of osteoporosis-related fractures, there is a growing interest in identifying genetic markers associated with osteoporosis in order to develop genetic screening strategies. To date, most candidate gene and genome-wide association studies (GWAS) examining the genetic factors influencing bone phenotype parameters have been focused on BMD assessed by DXA $[9,10]$. One of the most powerful studies, a meta-analysis pooling five GWAS, revealed novel strong associations between genetic variants in ZBTB40 (zinc finger- and BTB domain-containing 40), SP7 (Osterix) (Sp7 transcription factor 7) and AKAP11 (A-kinase anchoring protein 11) genes and BMD at femoral neck and lumbar spine [9] that have been confirmed in further studies [11-16].

Quantitative ultrasound (QUS) is considered a valuable screening tool for assessing bone status in clinical practice [17]. Compared to dual X-ray absorptiometry (DXA), QUS offer more accessibility to the population since is a non-invasive, radiation-free, transportable and low-cost device. Calcaneal QUS provides information on the main constituents of bone health, bone quantity (bone mass) and bone quality (bone microarchitecture) [18]. In addition, a meta-analysis of prospective studies revealed that calcaneal QUS predicts the risk of different types of fracture independently of DXA [19].

Familial studies have reported that calcaneal QUS parameters are under strong genetic control, with a suggested heritability of $74 \%$ [20]. In spite of this fact, a scarce number of studies have examined genetic factors associated with ultrasonography bone parameters [21]. A few studies have confirmed that different genetic BMD factors such as WNT16 are also associated with QUS parameters [21-24]. However, it has not been investigated if ZBTB40, SP7 and AKAP11 are also associated with other skeletal traits such as calcaneal QUS.

On the other hand, peak bone mass (PBM), defined as the level of bone mass achieves at the end of the grow period, is known to be influenced by both genetic and environmental factors [25, 26]. Twins and family studies suggested that $60-80 \%$ of the variance of the PBM is genetically determined [27]. Since it has been reported that a $10 \%$ increase in PBM may reduce the risk of osteoporotic fractures in older adults by $50 \%$ [28], the achievement of PBM in early ages is especially important in the prevention of osteoporosis. Therefore, there is a growing interest in investigating the genetic variants that influence bone status at early ages through primary effects on growth.

On this context, to determine if the associations between ZBTB40 (rs7524102, rs6426749), SP7 (rs2016266) and AKAP11 (rs9533090) and BMD was specific for this bone trait, for the first time, we have tested for associations between these genetic markers and calcaneal QUS parameter in a population of young adults.

\section{Material and methods}

\section{Subject characteristics}

A cross-sectional study was conducted on 550 Spanish healthy individuals of European ancestry (381 females and 169 males, median age 20.46 22,69). A detailed medical history was obtained for all subjects. In particular, those with a history of bone disease, metabolic or endocrine diseases, hormonal contraceptive therapy on medication that could affect bone mass were excluded from the study. Signed informed consent was obtained from all participants individually, the study was approved by local ethics committees, and it was conducted in accordance with the Declaration of Helsinki.

\section{Bone assessment}

The CUBA clinical ultrasound bone densitometer (McCue Ultrasonic Limited, Compton, Winchester, UK) was used to perform the QUS measurements. Bone mass was assessed through broadband ultrasound attenuation parameter (BUA, $\mathrm{dB} / \mathrm{MHz}$ ) on the right calcaneus. The apparatus was calibrated on a daily basis using a physical phantom to control its long-term stability.

\section{Covariates}

Weight to the nearest $0.1 \mathrm{~kg}$ was recorded using a Body Composition Analyser (TANITA BC-418MA), and height was measured to the nearest $0.1 \mathrm{~cm}$ using a Harpenden stadiometer. Both measurements were taken without shoes on. The same trained research assistant performed all the measurements. Physical activity were determined using the self-administered International Physical Activity Questionnaire (IPAQ) [29]. Dietary calcium intake (DCI) was assessed using the 72-hour recall method, considering intakes on Thursday, Friday and Saturday. Food records were converted to nutrient intake using a computerized nutrient analysis program (Nutriber 1.1.5).

\section{SNP selection and genotyping}

Saliva samples for DNA extraction were collected from the study participants using the OG-500 Collection Kit (DNA Genotek Inc, Ontario, Canada). The DNA was isolated from the saliva samples according to the manufacturer's protocol. We selected SNPs in ZBTB40 (rs7524102, rs6426749), SP7 (rs2016266) and AKAP11 (rs9533090) as genetic markers based on their previously identified genome-wide strong association with lumbar spine 
and/or femoral neck BMD in a meta-analysis pooling five GWAS [9]. Genotyping was performed at the Genomic and Genotyping Unit in the GENyO Center (Pfizer-University of Granada-Junta de Andalucía Centre for Genomics and Oncological Research) using TaqMan SNP Genotyping Assays (Applied Biosystems, Foster City, CA). TaqMan SNP genotyping plates were custom designed including four predesigned genotyping assays for each of the four selected SNPs. Standard cycling conditions were used, as recommended by the manufacturer. Thermal cycling and fluorescence detection were performed using the QuantStudio 12K Flex Real-Time PCR System (Applied Biosystems). The average missing rate for the genotyping assays was $1.54 \%$, with a range from $1.27 \%$ to $1.81 \%$. To guarantee the accuracy of genotyping, duplicate samples and negative controls were included in all genotyping arrays, presenting $100 \%$ identical genotypes.

\section{Statistical analysis}

The Haploview software (Broad Institute of MIT and Harvard) was used to calculate allele frequencies and verify that the genotype data were in Hardy-Weinberg equilibrium (HWE). Linear regression analysis was used to analyze the relationships between the SNPs and calcaneus ultrasounds adjusted for age, sex, weight, height, physical activity, and calcium intake. The results are reported as a percentage change $(\beta)$ in the standard deviation (SD) with $95 \%$ confidence intervals (95\% $\mathrm{CI}$ ). The most conservative method (Bonferroni correction) was applied to correct for multiple comparisons. Values of less than 0.05 were considered statistically significant. The cut-off value for significance was set as $p=0.05 / 4=0.012$. All statistical analyses were performed using SPSS version 20.0 (SPSS, Chicago, IL, USA). Statistical power of the study was estimated using Quanto version 1.2 software (Department of Preventive Medicine, University of Southern California).

\section{Results}

The basic characteristics of the 550 study subjects are shown in Table 1 . The average BUA value for the total population was $82.22 \pm 26.67 \mathrm{~dB} / \mathrm{Mhz}$. This value was similar to that previously observed for young adults [30, 31]. Mean weight was $63.90 \pm 12.8863 .7 \mathrm{~kg}$; mean height was $1.67 \pm 0.08 \mathrm{~m}$. Calcium intake averaged $800.157 \pm 349.90 \mathrm{mg} /$ day. PA averaged $2863.63 \pm 3876.92 \mathrm{MET} / \mathrm{min}$.

The location, genotype, and allele frequency of the SNPs selected as genetic markers are listed in Table 2. The observed MAF of all the SNPs examined was comparable to those reported by the
European-CEU population panel. The genotype frequencies were as follows: rs7524102 (AA 67.5\%; AG; 27.5\%; GG 5.0\%), rs6426749 (GG 59.0\%; GC $37.0 \%$; CC 4.0\%), rs2016266 (AA 40.65\%; AG 43.4\%; GG $16.0 \%$ ) and rs9533090 (CC 33.5\%; CT 45.5\%; TT $21.0 \%)$. None of the SNPs failed the missingness test (genotyping $>0.05$ ) or frequency test (MAF $<0.01$ ) and were observed to be in HWE.

Table 1. Characteristics of the study population.

\begin{tabular}{ll}
\hline Characteristic & Means \pm SD \\
\hline $\mathrm{N}$ & 550 \\
Age (years) & $20.46 \pm 2.69$ \\
Height $(\mathrm{m})$ & $1.67 \pm .08$ \\
Weight $(\mathrm{kg})$ & $63.90 \pm 12.88$ \\
BMI $\left(\mathrm{kg} / \mathrm{m}^{2}\right)$ & $22.70 \pm 3.75$ \\
Calcium intake $(\mathrm{mg} /$ day) & $800.157 \pm 349.90$ \\
Physical activity $(\mathrm{MET} / \mathrm{min})^{*}$ & $2863.63 \pm 3876.92$ \\
Heel ultrasound BUA $(\mathrm{dB} / \mathrm{Mhz})$ & $82.22 \pm 26.67$ \\
\hline Data are shown as mean \pm SD. & \\
*The calculation of MET-hours is described in methods section. \\
BMI body mass index, BUA broadband ultrasound attenuation.
\end{tabular}

Table 2. Overview of the selected SNPs, minor allele frequency (MAF) and Hardy-Weinberg Equilibrium (HWE).

\begin{tabular}{lllllll}
\hline $\begin{array}{l}\text { Closet } \\
\text { gene }\end{array}$ & \multicolumn{2}{l}{ Locus SNP } & $\begin{array}{l}\text { Allele* } \\
\text { MAF in this } \\
\text { study }\end{array}$ & $\begin{array}{l}\text { MAF HapMap } \\
\text { CEU }\end{array}$ & $\begin{array}{l}\text { HWE } \\
\text { (p) }\end{array}$ \\
\hline ZBTB40 & $1 \mathrm{p} 36$ & rs7524102 & A/g & 0.18 & 0.18 & 0.07 \\
ZBTB40 & $1 \mathrm{p} 36$ & rs6426749 & c/G & 0.22 & 0.12 & 0.15 \\
SP7 & $12 q 13$ & rs2016266 A/g & 0.37 & 0.33 & 0.08 \\
AKAP11 & $13 q 14$ & rs9533090 t/C & 0.43 & 0.43 & 0.11 \\
\hline *Capital letter indicates the major allele and small letter indicates the minor allele.
\end{tabular}

Linear regression analysis revealed that rs7524102 and rs6426749 in ZBTB40, and rs9533090 in AKAP11 were significantly associated with the calcaneal QUS parameter after adjustments for age, sex, weight, height, physical activity, and calcium intake $(\mathrm{p}=0.038, \mathrm{p}=0.012$ and $\mathrm{p}=0.008$, respectively) (Table 3). After applying the Bonferroni correction for multiple testing $(p=0.012)$, only the association of rs9533090 in AKAP11 remained significant. For the rs2016266 SNP in SP7, no significant association was observed after adjusting for confounding variables (Table 3).

Table 3. Linear regression analysis results for the association of the selected SNPs with BUA.

\begin{tabular}{llll}
\hline Closet gene & SNP & Heel BUA $(\mathrm{Db} / \mathrm{MHz})$ & \\
\hline & & $\beta(95 \% \mathrm{CI})$ & $\mathrm{p}$ \\
\hline ZBTB40 & rs7524102 & $0.087(0.215,7.785)$ & 0.038 \\
ZBTB40 & rs6426749 & $0.107(1.171,9.230)$ & 0.012 \\
SP7 & rs2016266 & $0.16(-2.529,3.711)$ & 0.710 \\
AKAP11 & rs9533090 & $-0.117(-7.813 .-1.155)$ & $0.008^{*}$
\end{tabular}

Beta represents the regression coefficient

Adjusted for age, sex, weight, height, physical activity and calcium intake.

*Associations withstanding Bonferroni correction are indicated with asterisks. 


\section{Discussion}

This study involved a population of well-characterized young healthy Caucasian individuals in which the association between ZBTB40, SP7 and AKAP11 genes and bone status assessed by calcaneal QUS were examined for the first time. Our results revealed that the rs 9533090 polymorphism in AKAP11 seems to be as a genetic factor influencing calcaneal QUS parameter during youth.

AKAP11 has been reported to be a gene conferring susceptibility to osteoporosis $[9,11,12,32$, 33]. In particular, the rs $9533090 \mathrm{SNP}$ has been associated with BMD assessed by DXA in large-scale meta-analysis of GWAS at the genome wide significant level $[9,11,12]$. In fact, this SNP reached the top $p$ value $\left(p=5.4 \times 10^{-25}\right)$ at lumbar spine BMD of the known loci identified by Rivadeneira et al. [9]. In this line, for the first time, in this study we identified a significant association of the rs9533090 SNP with the calcaneus ultrasound parameter even after applying Bonferroni correction $(\mathrm{p}=0.008)$. This SNP is located on chromosome 13, about $8 \mathrm{~Kb}$ upstream of AKAP11 (A kinase anchor protein 11 ) and $35 \mathrm{~Kb}$ downstream of DGKH (diacylglycerol kinase, eta). AKAP11 codifies for a protein expressed at high levels through spermatogenesis and in mature sperm that binds to protein kinase A [34]. A recent work in which gene expression profile of AKAP11 was examined in primary mouse osteoblasts undergoing differentiation, identified a constant expression during osteoblast maturation [32]. Hence, impaired signaling through this receptor due to genetic variation in the AKAP11 gene might exert a role in bone mass regulation. To date, the functional relevance of the rs9533090 SNP has not been investigated. Considering its location upstream of the gene, it could be hypothesized that this polymorphism may interfere the activity of regulatory elements that control the function of the AKAP11 gene. Another possibility that should be considered is that the rs9533090 SNP might be in linkage disequilibrium with the real causing variant. Thus, to fully characterize the molecular mechanisms underlying the association of AKAP11 gene with bone phenotypes, further studies are required to identify the real causing variant and its influence in the gene activity or expression.

On the other hand, we did not observe evidence of association at any of the other loci investigated (rs7524102 and rs6426749 in ZBTB40 gene and rs2016266 in SP7), and calcaneal QUS after Bonferroni correction. Thus, according to our findings it could be suggested that they do not play a major role in bone status during early adulthood. Previous candidate gene association, GWAS and meta-analysis of GWAS studies investigating these genetic markers have reported significant associations with BMD at different skeletal sites in European and Asian populations $[9,16,35-40]$. Based on these observations, and in previous findings from our and other researchers it may possible to hypothesize that some genetic markers for QUS and BMD may be the same, while others would be different. For example, previous evidence have shown that $V D R$ genetic markers are associated with BMD [41, 42] but no for calcaneal ultrasound parameters [21, 43]. Contrary, WNT16 gene has demonstrated to influence both BMD [24] and QUS [21-23]. In addition, the observed differences may have been due at least in part to age differences, given that most previous work have conducted among older adults, mainly postmenopausal women [35, 37, 38] or mixed population range in age from 18 to 96 years [9], and our study comprised a well-characterized cohort of young adults.

This study has some limitations; one is that the cross-sectional design does not allow determination of a causal relationship. In addition, it is difficult to completely exclude the possibility that the lack of association observed in ZBTB40 and SP7 SNPs could likely be due to inadequate power (i.e., modest sample size). Power calculations showed that, considering the BUA mean observed in our population (82.22 \pm 26.67$)$, MAFs of $0.18-0.44,550$ individuals, and a $5 \%$ type I error threshold, there was $50 \%$ power to detect increments in the mean using an additive genetic model. Therefore, further studies conducted among larger samples sizes of young adults would be useful to confirm our preliminary results. Another limitation of the present study is that since we looked at only four SNPs that had been previously associated with BMD, we did not cover all the genetic variation in the selected genes. Hence, other regions of these genes might be involved in regulating calcaneal QUS in early adulthood.

In conclusion, our study has provided evidence the AKAP11 gene (rs9533090) influences QUS trait in a population of Caucasian young adults. The rs9533090 SNP may be considered a factor affecting peak bone mass acquisition. This advance in knowledge about the genetic markers with which to assess bone status at early ages is crucial to optimize bone health and prevent osteoporosis later in life. Since this study is the first to investigate the associations between genetic markers in ZBTB40, SP7 and AKAP11 genes and calcaneal QUS, these findings require replication in independent populations. If confirmed, further molecular studies molecular studies are warranted to understand the underlying mechanisms responsible for this association. 


\section{Acknowledgments}

This study was supported by grant PI-0414-2014 from Consejería de Salud (Junta de Andalucía).

\section{Author Contributions}

Correa-Rodríguez M monitored data collection, wrote the statistical analysis plan, cleaned and analyzed the data and drafted and revised the paper. Schmidt-RioValle J and Rueda-Medina B analyzed the data, and drafted and revised the paper.

\section{Human and Animal Rights and Informed Consent}

All procedures performed in studies involving human participants were in accordance with the ethical standards of the institutional and/or national research committee and with the 1964 Helsinki declaration and its later amendments or comparable ethical standards.

\section{Competing Interests}

The authors have declared that no competing interest exists.

\section{References}

1. NIH Consensus Development Panel on Osteoporosis Prevention, Diagnosis, and Therapy. Osteoporosis prevention, diagnosis, and therapy. JAMA. 2001;285:785-95.

2. Reginster J-Y, Burlet N. Osteoporosis: A still increasing prevalence. Bone. 2006;38:4-9.

3. Adachi JD, Adami S, Gehlbach S, et al. Impact of prevalent fractures on quality of life: baseline results from the global longitudinal study of osteoporosis in women. Mayo Clin Proc. 2010;85:806-13.

4. Johnell O, Kanis JA. An estimate of the worldwide prevalence and disability associated with osteoporotic fractures. Osteoporos Int. 2006;17:1726-1733.

5. Burge R, Dawson-Hughes $\mathrm{B}$, Solomon $\mathrm{DH}$, et al. Incidence and economic burden of osteoporosis-related fractures in the United States, 2005-2025. J Bone Miner Res. 2007;22:465-475.

6. Sigurdsson G, Halldorsson B V, Styrkarsdottir U, et al. Impact of genetics on low bone mass in adults. J Bone Miner Res. 2008;23:1584-90.

7. Clark GR, Duncan EL. The genetics of osteoporosis. Br Med Bull. 2015; 113:73-81.

8. Burke W, Tarini B, Press NA, Evans JP. Genetic Screening. Epidemiol Rev. 2011;33:148-164.

9. Rivadeneira F, Styrkársdottir U, Estrada K, et al. Twenty bone-mineral-density loci identified by large-scale meta-analysis of genome-wide association studies. Nat Genet. 2009;41:1199-206.

10. Estrada K, Styrkarsdottir U, Evangelou E. Genome-wide meta-analysis identifies 56 bone mineral density loci and reveals 14 loci associated with risk of fracture. Nat Genet. 2012;44:491-501.

11. Lill CM, Liu T, Norman K, et al. Genetic burden analyses of phenotypes relevant to aging in the Berlin Aging Study II (BASE-II). Gerontology. 2016;62:316-322.

12. Zhang L, Choi HJ, Estrada K, et al. Multistage genome-wide association meta-analyse identified two new loci for bone mineral density. Hum Mol Genet. 2014;23:1923-33.

13. Park SE, Oh KW, Lee WY, et al. Association of osteoporosis susceptibility genes with bone mineral density and bone metabolism related markers in Koreans: The Chungju Metabolic Disease Cohort (CMC) study. Endocr J. 2014;61:1069-1078.

14. Styrkarsdottir U, Halldorsson B V, Gudbjartsson DF, et al. European bone mineral density loci are also associated with BMD in East-Asian populations. PLoS One. 2010;5:e13217.

15. Liu J, Zhang M, Zhao L, et al. Analysis of recently identified osteoporosis susceptibility genes in Han Chinese women. J Clin Endocrinol Metab. 2010;95:E112-20.
16. Timpson NJ, Tobias JH, Richards JB, et al. Common variants in the region around Osterix are associated with bone mineral density and growth in childhood. Hum Mol Genet. 2009;18:1510-1517.

17. Hans D, Baim S. Quantitative ultrasound (QUS) in the management of osteoporosis and assessment of fracture risk. J Clin Densitom. 2017;20:322-333.

18. Chin K-Y, Ima-Nirwana S. Calcaneal quantitative ultrasound as a determinant of bone health status: what properties of bone does it reflect? Int J Med Sci. 2013;10:1778-83.

19. Moayyeri A, Adams JE, Adler RA, et al. Quantitative ultrasound of the heel and fracture risk assessment: an updated meta-analysis. Osteoporos Int. 2012;23:143-53

20. Karasik D, Hsu YH, Zhou Y, et al. Genome-wide pleiotropy of osteoporosis-related phenotypes: The Framingham study. J Bone Miner Res. 2010;25:1555-1563.

21. Moayyeri A, Hsu Y-H, Karasik D, et al. Genetic determinants of heel bone properties: genome-wide association meta-analysis and replication in the GEFOS/GENOMOS consortium. Hum Mol Genet. 2014;23:3054-3068

22. García-Ibarbia C, Pérez-Núñez MI, Olmos JM, et al. Missense polymorphisms of the WNT16 gene are associated with bone mass, hip geometry and fractures. Osteoporos Int. 2013;24:2449-2454.

23. Correa-Rodríguez M, Schmidt-RioValle J, Rueda-Medina B. Polymorphisms of the WNT16 gene are associated with the heel ultrasound parameter in young adults. Osteoporos Int. 2016;27:1057-1061.

24. Zheng HF, Tobias JH, Duncan E, et al. WNT16 influences bone mineral density, cortical bone thickness, bone strength, and osteoporotic fracture risk. PLoS Genet. 2012;8:e1002745.

25. Bonjour J-P, Chevalley $\mathrm{T}$, Ferrari S, Rizzoli R. The importance and relevance of peak bone mass in the prevalence of osteoporosis. Salud Publica Mex. 2009; 51:s5-s17.

26. Lu J, Shin Y, Yen M-S, Sun SS. Peak bone mass and patterns of change in total bone mineral density and bone mineral contents from childhood into young adulthood. J Clin Densitom. 2016;19:180-91.

27. Heaney RP, Abrams S, Dawson-Hughes B, et al. Peak bone mass. Osteoporos Int. 2001;11:985-1009.

28. Cummings SR, Black DM, Nevitt MC, et al. Bone density at various sites for prediction of hip fractures. The Study of Osteoporotic Fractures Research Group. Lancet (London, England). 1993;341:72-5.

29. Craig CL, Marshall AL, Sjostrom M, et al. International physical activity questionnaire: 12-country reliability and validity. Med Sci Sport Exerc. $2003 ; 35: 1381-1395$

30. Scheffler C, Gniosdorz B, Staub K, Rühli F. Skeletal robustness and bone strength as measured by anthropometry and ultrasonography as a function of physical activity in young adults. Am J Hum Biol. 2014; 26:215-220.

31. Babaroutsi E, Magkos F, Manios Y, Sidossis LS. Lifestyle factors affecting heel ultrasound in Greek females across different life stages. Osteoporos Int. 2005;16:552-561.

32. Nielson CM, Liu C-T, Smith A V, et al. Novel genetic variants associated with increased vertebral volumetric BMD, reduced vertebral fracture risk, and increased expression of SLC1A3 and EPHB2. J Bone Miner Res. 2016;31:2085-2097.

33. Roshandel D, Thomson W, Pye SR, et al. A validation of the first genome-wide association study of calcaneus ultrasound parameters in the European Male Ageing Study. BMC Med Genet. 2011;12:1-9.

34. Duncan EL, Brown MA. Genetic determinants of bone density and fracture risk--state of the art and future directions. J Clin Endocrinol Metab. 2010; 95:2576-2587.

35. Park SE, Oh KW, Lee WY, et al. Association of osteoporosis susceptibility genes with bone mineral density and bone metabolism related markers in Koreans: The Chungju Metabolic Disease Cohort (CMC) study. Endocr J. 2014;61:1069-1078.

36. Liu JM, Zhang MJ, Zhao L, et al. Analysis of recently identified osteoporosis susceptibility genes in Han Chinese women. J Clin Endocrinol Metab. 2010; 95:112-120.

37. Styrkarsdottir U, Halldorsson B V., Gudbjartsson DF, et al. European bone mineral density loci are also asociated with BMD in East-Asian populations. PLoS One. 2010;5:e13217.

38. Styrkarsdottir U, Halldorsson B V., Gretarsdottir S, et al. Multiple genetic loci for bone mineral density and fractures. $N$ Engl J Med. 2008;358:2355-2365

39. Styrkarsdottir U, Halldorsson B V, Gretarsdottir S, et al. New sequence variants associated with bone mineral density. Nat Genet. 2009;41:15-17.

40. Ho-Pham LT, Nguyen SC, Tran B, Nguyen T V. Contributions of Caucasian-associated bone mass loci to the variation in bone mineral density in Vietnamese population. Bone. 2015;76:18-22. 
41. Canto-Cetina T, Cetina Manzanilla JA, González Herrera L, et al. VDR polymorphisms are associated with bone mineral density in post-menopausal Mayan-Mestizo women. Ann Hum Biol. 2015;42:470-5.

42. Wang D, Liu R, Zhu H, et al. Vitamin D receptor Fok I polymorphism is associated with low bone mineral density in postmenopausal women: a meta-analysis focused on populations in Asian countries. Eur J Obstet Gynecol Reprod Biol. 2013;169:380-6.

43. Correa-Rodríguez M, Schmidt Rio-Valle J, González-Jiménez E, Rueda-Medina B. A cross-sectional study of the association of VDR gene, calcium intake, and heel ultrasound measures in early adulthood. Calcif Tissue Int. 2016; 98:226-34. 